\title{
Germanica
}

\section{Le « petit » roman de Brigitte Burmeister}

\section{Nicole Bary}

\section{OpenEdition}

Journals

Édition électronique

URL : http://journals.openedition.org/germanica/2636

DOI : 10.4000/germanica.2636

ISSN : 2107-0784

\section{Éditeur}

Université de Lille

\section{Édition imprimée}

Date de publication : 31 décembre 1989

Pagination : 167-179

ISSN : 0984-2632

\section{Référence électronique}

Nicole Bary, «Le « petit » roman de Brigitte Burmeister », Germanica [En ligne], 5 | 1989, mis en ligne le 10 février 2015, consulté le 06 octobre 2020. URL : http://journals.openedition.org/germanica/2636 ; DOI : https://doi.org/10.4000/germanica.2636

Ce document a été généré automatiquement le 6 octobre 2020.

(c) Tous droits réservés 


\section{Le « petit » roman de Brigitte Burmeister}

Nicole Bary

\section{RÉFÉRENCE}

Brigitte Burmeister est née en 1940 à Poznan. Après des études de langues et littératures romanes à Leipzig, elle a travaillé à l'Académie des Sciences de R.D.A. et participé à des recherches sur la littérature française, en particulier la littérature contemporaine. En 1983, elle a publié un essai Streit um den Nouveau Roman et en 1986 une étude sur la littérature des femmes Weibliches Schreiben. Zu einigen Aspekten französischer Frauentexte in den siebziger Jahren. Depuis 1983, Brigitte Burmeister n'a plus de fonction universitaire, elle est traductrice. Avec ce « petit » roman, elle a fait ses débuts littéraires.

1 Chaque littérature cultive les genres littéraires issus de son patrimoine culturel, chaque époque a son esthétique et sa sensibilité. Il semble que le roman soit un genre un peu délaissé par les écrivains contemporains de langue allemande. La plupart des auteurs renoncent d'ailleurs à indiquer le genre, les plus hardis se risquant à mentionner qu'il s'agit de prose. L'œuvre de Brigitte Burmeister, dont nous présentons des extraits en traduction, défie cette constatation. L'auteur la caractérise elle-même, comme un « petit» roman ${ }^{1}$.

Petit, par rapport à qui, à quoi ? Peut-on avancer que cette épithète est sans doute un hommage de l'auteur à ses maîtres, Kafka, Borges, les pères fondateurs du nouveau roman français - Claude Simon ou Alain Robbe-Grillet voire même les écrivains de la seconde génération? Certaines pages du roman renvoient très clairement à Le Clézio. Rien d'étonnant d'ailleurs. L'auteur est romaniste et à consacré plusieurs études au nouveau roman français. Mais ici, les affinités ne sont pas seulement intellectuelles, elles concernent aussi la sensibilité, l'esthétique, l'écriture. 
3 Le titre volontairement ambigu Anders oder Vom Aufenthalt in der Fremde suggère la thématique autour de laquelle s'articule le roman. David Anders, le narrateur, se définit à la fois par sa non-relation aux autres et par son désir irrépressible d'être un autre. La problématique du personnage en quête d'identité est d'ailleurs soulignée par la seconde partie du titre Vom Aufenthalt in der Fremde. La juxtaposition du nom du narrateur Anders - et du mot "Fremde ", l'étranger, indique les intentions de l'auteur : toute situation nouvelle, qui n'est pas familière, permet non seulement la perception d'une autre image de la réalité, mais aussi la prise de conscience par le sujet qui l'observe d'un côté jusque-là inconnu de son identité.

4 Dans les cinquante-trois séquences qui forment le livre, David Anders regarde un monde inconnu, étranger, la grande ville, Berlin sans aucun doute - quelques allusions à la ville partagée sont limpides -, mais l'auteur ne donne aucune précision sur ce point. Dans des lettres - sont-ce bien des lettres, les envoie-t-il à leurs destinataires, à ceux qui lui sont « chers » et qui sont restés à la maison ? - il consigne ses observations. Son œil, tel une de ces anciennes caméras que l'on utilisait autrefois pour réaliser des films fixes, fige le réel dans un applat qui suggère ennui et monotonie. Il a le regard froid et impénétrable d'un chat sauvage. L'œil n'est qu'un instrument de travail qui exclue tout contact avec la réalité, il se contente de décoder le spectacle qui s'offre à sa vue ou de le contempler comme un tableau abstrait. Le lecteur, à la poursuite du narrateur s'engage dans le labyrinthe de ces cinquante-trois chapitres et avance au gré du rythme ample de l'écriture de Brigitte Burmeister.

5 Dans le roman, c'est par rapport au narrateur que s'organisent les différentes couches du réel dans leurs relations les unes aux autres. Le moi narratif assume dans l'œuvre diverses fonctions. Il permet, entre autres à l'auteur, de formuler les rapports réciproques entre la vie et l'écriture.

6 David Anders est un fonctionnaire sans famille, sans relations, sans obligations, taillable et corvéable à merci. Il est, au début du roman, l'incarnation de la perception pure, de l'observation objective qui ne se laisse pas pervertir par sa subjectivité. Mais ce sont précisément les règles que lui impose sa fonction - la distance et le détachement nécessaires à l'observation impartiale - qui lui révèlent sa puissance créatrice potentielle. Au fur et à mesure que les semaines passent, ses comptes rendus hebdomadaires se modifient. Les observations laissent peu à peu la place à des souvenirs, à des évocations, à des allusions. Le Moi profond remonte, s'impose, David Anders passe insensiblement de l'observation du monde extérieur à l'exploration de son monde intérieur. Les procès-verbaux des premières semaines se transforment. Les récits s'articulent tantôt sur le vécu et la mémoire du narrateur, tantôt sur un jeu de rôles qui sont l'expression des différentes facettes de son identité brisée. Les cinquantedeux semaines de son séjour dans la ville inconnue sont le prétexte à une excursion dans les zones cachées et donc étrangères de son Moi.

7 L'histoire de David Anders est aussi une réflexion sur l'art et la société, sur l'artiste dans la société. Anders à l'affût de la réalité, l'artiste voyeur, ce ne sont pas là des thèmes neufs pour la littérature. Le contexte dans lequel se situe le roman de Brigitte Burmeister, lui confère, par contre, une dimension peu présente jusque là dans la littérature de la R.D.A. : les rapports de l'art et de la société dans un pays socialiste. La rencontre fortuite d'Anders avec une femme - elle est écrivain - et son compagnon l'introduit dans un univers qui se joue du pouvoir quel qu'il soit. L'art, la littérature ne reconnaissent que les règles internes qu'ils se donnent à eux-mêmes, refusant la 
subordination au pouvoir. En ce sens le roman de Brigitte Burmeister apporte une réponse très personnelle à la question maintes fois posée à propos de la littérature de la R.D.A. - la fonction sociale qu'occupe la littérature dans le pays est-elle un bien pour la littérature ? - et il s'insère fort à propos dans le débat qui anime les écrivains de R.D.A. depuis quelque temps et dont le dernier congrès de l'Union des Écrivains a été la tribune.

8 L'alliance des réflexions sur l'art et sur la société est aussi un élément de l'architecture interne de l'ensemble des chapitres du roman. Les observations, puis les impressions ou les évocations sont la matière sur laquelle le narrateur/l'auteur articule sa réflexion. Ce corpus de textes forme une chronique homogène qui exprime à la fois l'histoire d'une conscience et l'image figée d'une réflexion.

Le roman de David Anders est finalement l'histoire d'une conscience qui s'affranchit de la société en découvrant que l'art est l'instance qui rend possible la résistance à la société. L'histoire de David Anders est l'histoire d'un homme et de son double. David Anders est à la fois David et Goliath, il est l'instrument au service de l'Histoire et l'homme libre, le créateur qui sait rester critique de l'Histoire. Le roman de Brigitte Burmeister est une spirale sans fin. Le séjour à l'étranger ne prend pas fin avec le retour d'Anders à la maison. Anders sait maintenant que l'étranger est en lui, qu'il est l'un et l'autre.

\section{NOTES}

1. Anders oder Vom Aufenthalt in der Fremde. Ein kleiner Roman, Verlag der Nation, Berlin-R.D.A. et Luchterhand, Darmstadt, 1988. 